\title{
Structural Insights into the Catalytic Mechanism of Granzyme B upon Substrate and Inhibitor Binding
}

\author{
Neha Tripathi ${ }^{1}$, Richard Danger ${ }^{1}$, Mélanie Chesneau ${ }^{1}$, Sophie Brouard ${ }^{1}$, and Adèle \\ Laurent $^{1}$ \\ ${ }^{1}$ Université de Nantes
}

January 19, 2021

\begin{abstract}
Human granzyme B (hGzmB), which is present in various immune cells, has attracted much attention due to its role in various pathophysiological conditions. The hGzmB activity is triggered at a catalytic triad (His59, Asp103, Ser198), cleaving its specific substrates. To date, the drug design strategy against hGzmB mainly targets the catalytic triad, which causes the non-specificity problem of inhibitors due to the highly conserved active site in serine proteases. In the present work, microsecond classical molecular dynamics simulations are devoted to exploring the structural dynamics of the hGzmB catalytic cycle in the presence of Ac-IEPD-AMC, a known substrate (active hGzmB), and Ac-IEPD-CHO, a known inhibitor (inactive hGzmB). By comparing active and inactive forms of hGzmB in the six different stages of the hGzmB catalytic cycle, we revealed, for the very first time, an additional network of interactions involving Arg216, a residue located outside the conventional binding site. Upon activation, the His59[?][?][?]Asp103 hydrogen bond is broken due to the formation of the Asp103[?][?][?]Arg216 salt bridge, expanding the active site to facilitate the substrate-binding. On the contrary, the binding of inhibitor Ac-IEPD-CHO to hGzmB prevents the Arg216-mediated interactions within the catalytic triad, thus preventing hGzmB activity. In silico Arg216Ala mutation confirms the role of Arg216 in enzyme activity, as the substrate Ac-IEPD-AMC failed to bind to the mutated hGzmB. Importantly, as Arg216 is not conserved amongst the various granzymes, the current findings can be a major step to guide the design of hGzmB specific therapeutics.
\end{abstract}

\section{Hosted file}

Manuscript-Proteins-07-01-2021.pdf available at https://authorea.com/users/390344/articles/ 504712-structural-insights-into-the-catalytic-mechanism-of-granzyme-b-upon-substrateand-inhibitor-binding 